\title{
Dynamic Inventory Management with Cash Flow Constraints
}

\author{
Xiuli Chao, ${ }^{1}$ Jia Chen, ${ }^{2}$ Shouyang Wang ${ }^{2}$ \\ ${ }^{1}$ Department of Industrial and Operations Engineering, University of Michigan, Ann Arbor, Michigan 48109-2117 \\ ${ }^{2}$ Institute of Systems Science, Chinese Academy of Sciences, Beijing 100080, China
}

Received 6 June 2007; revised 3 August 2008; accepted 9 August 2008

DOI 10.1002/nav.20322

Published online 21 October 2008 in Wiley InterScience (www.interscience.wiley.com).

\begin{abstract}
In this article, we consider a classic dynamic inventory control problem of a self-financing retailer who periodically replenishes its stock from a supplier and sells it to the market. The replenishment decisions of the retailer are constrained by cash flow, which is updated periodically following purchasing and sales in each period. Excess demand in each period is lost when insufficient inventory is in stock. The retailer's objective is to maximize its expected terminal wealth at the end of the planning horizon. We characterize the optimal inventory control policy and present a simple algorithm for computing the optimal policies for each period. Conditions are identified under which the optimal control policies are identical across periods. We also present comparative statics results on the optimal control policy. () 2008 Wiley Periodicals, Inc. Naval Research Logistics 55: 758-768, 2008
\end{abstract}

Keywords: dynamic inventory management; financial constraint; base-stock policy

\section{INTRODUCTION}

The majority of the current literature on inventory management ignores financial status of a firm and assumes that the firm is able to implement any replenishment and ordering decisions, see, $[2,14,15,19]$. On the other hand, it is well-known that cash flow is one of the key reasons for the bankruptcy of small- and medium-sized companies. For example, Bradley [5] surveys 531 businesses in the Southwest United States that went bankrupt during the calendar year 1998, and the study suggests that inadequate financial planning, especially operating capital for the early months of the operation, is a most evident factor for bankruptcy. Another study was conducted by a research group in the Development and Research Center of State Council of China, see Chen and Zhang [7], where similar findings are reported.

In this article, we consider a classic dynamic inventory control problem of a self-financing retailer who periodically replenishes its stock from a supplier and sells it to the market. Excess demand in each period is lost when insufficient inventory is in stock. The demands for different periods are independent and identically distributed random variables. The retailer's operational decisions are constrained by its cash flow, which is updated periodically following purchasing and sales in each period. We seek to gain understanding on how

Correspondence to: X. Chao (xchao@umich.edu) operational decisions interact with and are affected by cash flows in a dynamic setting. The objective of the firm is to maximize its expected wealth level at the end of the planning horizon. We obtain the explicit structure on how the optimal inventory control strategy depends on the cash flows. We also study the relationship between the optimal control policy and the system parameters, e.g., purchasing price, interest rate, savage value, and selling price. Conditions are identified under which the optimal control policies are identical across periods. A simple algorithm is developed to compute the optimal inventory control policy for each period. Though in the article we focus on lost-sales model, similar result can be obtained for the backlog case.

There are several papers that deal with budgetary constraints. By assuming the availability of market hedges, Birge [4] adopts option pricing theory for incorporating risk into planning models by adjusting capacity and resource levels. Rosenblatt and Rothblum [16] treat capacity as a decision variable in their study of multi-item inventory systems under a single resource capacity constraint. Li et al. [13] consider a single-product firm that makes production decisions, borrowing decisions and dividend policies for each period while facing uncertain demand. The firm maximizes the expected present value of the infinite-horizon flow of the dividends subjecting to loan size, production size, and liquidity constraints. The firm can obtain an unbounded single-period loan with a constant interest rate. The authors derive the 
optimal myopic policies and study their structural properties. Archibald et al. [1] consider a start-up firm facing discretely distributed demand and the objective is to maximize the long-term survival probability instead of average profit per period. The authors conclude that start-up firms should be more cautious in their component purchasing strategy than well-established firms. They also show that the strategy is not monotone in the amount of capital available. Babich and Sobel [3] study the coordination of financial decisions (loan size) and operational decisions (production and sales) to maximize the expected discounted proceeds from an initial public offering (IPO). They model the IPO event as a stopping time in an infinite-horizon discounted Markov decision process. Furthermore, they characterize an optimal capacity-expansion policy and obtain sufficient conditions for a monotone threshold rule to yield an optimal IPO decision. Hu and Sobel [12] study a dynamic newsvendor model with the criterion of maximizing the expected present value of dividends, and examine the interdependence of a firm's capital structure and its short-term operating decisions concerning inventories, dividends, and liquidity. They obtain interesting results on the interaction between firm's capital structure and operational decisions. The work that also addresses the interface of inventory management and finance is Buzacott and Zhang [6] who analyze a Stackelberg game between the bank and the retailer in a newsvendor inventory model. Buzacott and Zhang consider a single period inventory management problem where the bank's decisions include the interest rate to charge and the loan limit, and the retailer needs to decide the amount to borrow within the loan limit and the amount of inventory to order from suppliers. Both the bank and retailer maximize their expected returns. Other related work in this area includes Xu and Birge [18], and the references therein. In contrast to Buzacott and Zhang [6], in this article we consider a multiperiod dynamic model. However, we only focus on the retailer and do not consider the game between the bank and the retailer.

In a sense, financial constraint in inventory management can be considered as a supply capacity constraint on ordering quantity. Production-inventory problems with supply capacity constraints have received a great amount of attention since the work of $[9,10]$, see, e.g., $[8,17]$ among others. The main result for such systems is that the optimal control strategy is a modified base-stock policy. That is, there exists an optimal base-stock level, which is greater than or equal to that for the case without capacity constraint, and the optimal policy always tries to bring the inventory to the base-stock level; in case that cannot be achieved due to capacity constraint, then order as much as possible. The major difference between these two classes of models lies in the fact that in inventory control problems with supply capacity constraints, the constraints are given externally, whereas in inventory models with financial constraints, the financial constraints are the result of the firm's past decisions. Therefore, the financial constraints are themselves decisions. As a result, in making inventory decisions, its impact on future financial flows has to be taken into consideration.

The rest of this article is organized as follows. Section 2 presents the model and results. Some numerical studies are also included in Section 2. The article concludes in Section 3 with some remarks and some possible extensions. Throughout the article we use "increasing" and "decreasing" in nonstrict sense, i.e., they represent "nondecreasing" and "nonincreasing," respectively. For convenience, for any real number $x$, we denote $x^{+}=\max \{x, 0\}$.

\section{MODEL AND RESULTS}

We consider the periodic-review inventory control problem where a self-financing retailer sells a single product to the market. The risk neutral retailer faces random demand and makes replenishment decisions over a finite planning horizon of $N$ periods. The periods are numbered 1 to $N$, i.e., the first period is 1 , and the last period is $N$. The successive periods' demands $D_{n}, 1 \leq n \leq N$, are independent and identically distributed nonnegative random variables, with $f(\cdot)$ and $F(\cdot)$ being their probability density and cumulative distribution functions, respectively. We focus on a lost-sales model in this article, that is, unmet demand in each period is lost when insufficient inventory is in stock. The ordering lead time is zero.

Let $p$ be the unit selling price, and $c$ the unit ordering cost. Any inventory left at the end of the planning horizon has a salvage value $\gamma$ per unit. Surplus capital in each period is deposited in a savings account to earn an interest rate $d$ per period. To avoid triviality we assume

$$
-\infty<\gamma \leq c<p,
$$

with a negative value of $\gamma$ representing disposal cost. We further assume $(1+d) c<p$. If this condition is not satisfied, then the firm would always prefer to have all its capital in the banking account.

The sequence of events in each period is as follows. At the beginning of each period, the retailer places an order with its capital on hand, and deposits the surplus capital in the savings account. During the period demand is realized. At the end of the period the retailer receives its revenue from sales and savings interest.

Let $S_{n}$ be the capital level at the beginning of period $n$, let $x_{n}$ and $y_{n}$ be the inventory levels, before and after the replenishment decisions respectively, at the beginning of period $n$, and let $S_{N+1}$ be the terminal wealth at the end of the planning horizon.

Because the firm is self-financed, the ordering decision satisfies the cash flow constraint $c\left(y_{n}-x_{n}\right) \leq S_{n}$, and 
the remaining capital in period $n, S_{n}-c\left(y_{n}-x_{n}\right)$, is deposited in the savings account to generate an interest of $d\left(S_{n}-c\left(y_{n}-x_{n}\right)\right)$. The revenue from sales in period $n$ is $p \min \left\{y_{n}, D_{n}\right\}$. Hence the total capital level at the end of period $n$, which is also the capital level at the beginning of period $n+1$, is

$$
\begin{array}{r}
S_{n+1}=p \min \left\{y_{n}, D_{n}\right\}+(1+d)\left(S_{n}-c\left(y_{n}-x_{n}\right)\right), \\
n=1,2, \ldots, N .
\end{array}
$$

Because we consider lost-sales model, the inventory level at the beginning of period $n+1$ is

$$
x_{n+1}=\left(y_{n}-D_{n}\right)^{+}, \quad n=1,2, \ldots, N \text {. }
$$

Therefore, the decision problem of the retailer is to decide an ordering policy to maximize the expected terminal wealth at the end of the planning horizon, given initial inventory level $x_{1}$ and initial capital level $S_{1}$, subject to the cash flow constraint in each period. That is, the decision problem is

$$
\max _{y_{1}, \ldots, y_{N}} E\left[S_{N+1}\right]
$$

subject to (2), (3), and

$$
0 \leq y_{n}-x_{n} \leq S_{n} / c, \quad n=1,2, \ldots, N
$$

Denote by $V_{n}(x, S)$ the maximum expected terminal wealth given that the inventory level and capital level at the beginning of period $n$ are $x$ and $S$, respectively. The optimality equation is

$$
\begin{aligned}
V_{n}(x, S)= & \max _{x \leq y \leq x+S / c} E\left[V _ { n + 1 } \left(\left(y-D_{n}\right)^{+},\right.\right. \\
& \left.\left.p \min \left\{y, D_{n}\right\}+(1+d)(S-c(y-x))\right)\right],
\end{aligned}
$$

with a boundary condition

$$
V_{N+1}(x, S)=S+\gamma x .
$$

The trade-off in the dynamic programming equation above is between ordering inventory (and therefore earning profit from sales) and putting cash in savings account (and earning interests). When inventory is ordered, the retailer runs the risk of not selling the inventory and therefore loses the opportunity of earning an interest. Note that the problem in the final period is effectively a newsvendor problem with order quantity limit.

To derive the optimal control strategy, several lemmas are needed. The first lemma follows immediately from induction.

LEMMA 1: For any period $n$ and fixed $x, V_{n}(x, S)$ is increasing in $S$.
Lemma 1 is intuitively clear: The more initial capital the firm has, the better it is toward the firm's terminal wealth level.

To establish a second-order property of the value function $V_{n}$, we need the following result.

LEMMA 2: For any $n, V_{n}(A-z, B+p z)$ is increasing in $z$ for fixed $A$ and $B$.

PROOF: Note the relationship

$$
\begin{gathered}
V_{n}(A-z, B+p z)=\max _{A-z \leq y \leq A+B / c+(p-c) z / c} E\left[V _ { n + 1 } \left(\left(y-D_{n}\right)^{+},\right.\right. \\
\left.p \min \left\{y, D_{n}\right\}+(1+d)(c A+B+(p-c) z-c y)\right] .
\end{gathered}
$$

It follows from Lemma 1 that the function being maximized above is increasing in $z$. Because the feasible region $A-z \leq y \leq A+B / c+(p-c) z / c$ is also increasing in $z$, $V_{n}(A-z, B+p z)$ is increasing in $z$.

Lemma 2 is essential in proving the second-order property of the value function. The lemma says that it is better to keep cash than having inventory in stock at the beginning of the period. This can be intuitively explained as follows: Capital at the beginning of a period is more flexible than inventory in stock because the firm can always convert it to inventory by placing an order. However, the reverse is not true. In particular, if the on-hand inventory is higher than necessary, it would have been better to have part of that inventory in the form of cash to earn interest.

LEMMA 3: For any $n, V_{n}(x, S)$ is jointly concave in $x$ and $S$.

PROOF: We prove the lemma by backward induction. Clearly, $V_{N+1}(x, S)=S+\gamma x$ is jointly concave in $x$ and $S$. Assume that $V_{n+1}(x, S)$ is jointly concave in $x$ and $S$. We now prove the property for $n$.

We firstly prove $V_{n+1}\left(\left(y-D_{n}\right)^{+}, p \min \left\{y, D_{n}\right\}+(1+\right.$ d) $(S-c(y-x)))$ is jointly concave in $(y, x, S)$. For any $\left(y_{1}, x_{1}, s_{1}\right)$ and $\left(y_{2}, x_{2}, S_{2}\right)$ and $0 \leq \lambda \leq 1$, we need to prove

$$
\begin{aligned}
& V_{n+1}\left(\left(\lambda y_{1}+(1-\lambda) y_{2}-D_{n}\right)^{+}, p \min \left\{\lambda y_{1}+(1-\lambda) y_{2}, D_{n}\right\}\right. \\
& \quad+(1+d)\left(\lambda S_{1}+(1-\lambda) S_{2}-c\left(\lambda y_{1}+(1-\lambda)\right.\right. \\
& \left.\left.\left.\quad y_{2}-\lambda x_{1}-(1-\lambda) x_{2}\right)\right)\right) \\
& \leq \\
& \quad \lambda V_{n+1}\left(\left(y_{i}-D_{n}\right)^{+}, p \min \left\{y_{1}, D_{n}\right\}\right. \\
& \left.\quad+(1+d)\left(S_{1}-c\left(y_{1}-x_{1}\right)\right)\right) \\
& \quad+(1-\lambda) V_{n+1}\left(\left(y_{2}-D_{n}\right)^{+}, p \min \left\{y_{2}, D_{n}\right\}\right. \\
& \left.\quad+(1+d)\left(S_{2}-c\left(y_{2}-x_{2}\right)\right)\right) .
\end{aligned}
$$


Note the relationship $\left(y-D_{n}\right)^{+}=y-\min \left\{y, D_{n}\right\}$. For convenience let

$$
\begin{aligned}
& \bar{y}=\lambda y_{1}+(1-\lambda) y_{2}, \\
& \tilde{y}=\min \left\{\lambda y_{1}+(1-\lambda) y_{2}, D_{n}\right\} \\
& \hat{y}=\lambda \min \left\{y_{1}, D_{n}\right\}+(1-\lambda) \min \left\{y_{2}, D_{n}\right\} .
\end{aligned}
$$

Then by $\tilde{y} \geq \hat{y}$, we have

$$
\begin{aligned}
V_{n+1}( & \left(\lambda y_{1}+(1-\lambda) y_{2}-D_{n}\right)^{+}, \\
& p \min \left\{\lambda y_{1}+(1-\lambda) y_{2}, D_{n}\right\} \\
& +(1+d)\left(\lambda S_{1}+(1-\lambda) S_{2}-c\left(\lambda y_{1}+(1-\lambda) y_{2}\right.\right. \\
& \left.\left.\left.-\lambda x_{1}-(1-\lambda) x_{2}\right)\right)\right) \\
= & V_{n+1}\left(\bar{y}-\tilde{y}, p \tilde{y}+(1+d)\left(\lambda S_{1}+(1-\lambda) S_{2}\right.\right. \\
& \left.\left.-c\left(\lambda y_{1}+(1-\lambda) y_{2}-\lambda x_{1}-(1-\lambda) x_{2}\right)\right)\right) \\
\geq & V_{n+1}\left(\bar{y}-\hat{y}, p \hat{y}+(1+d)\left(\lambda S_{1}+(1-\lambda) S_{2}\right.\right. \\
& \left.\left.-c\left(\lambda y_{1}+(1-\lambda) y_{2}-\lambda x_{1}-(1-\lambda) x_{2}\right)\right)\right) \\
= & V_{n+1}\left(\lambda\left(y_{1}-D_{n}\right)^{+}+(1-\lambda)\left(y_{2}-D_{n}\right)^{+},\right. \\
& \lambda p \min \left\{y_{1}, D_{n}\right\}+(1-\lambda) p \min \left\{y_{2}, D_{n}\right\} \\
& +(1+d)\left(\lambda S_{1}+(1-\lambda) S_{2}-c\left(\lambda y_{1}+(1-\lambda) y_{2}\right.\right. \\
& \left.\left.\left.-\lambda x_{1}-(1-\lambda) x_{2}\right)\right)\right) \\
\geq & \lambda V_{n+1}\left(\left(y_{1}-D_{n}\right)^{+}, p \min \left\{y_{1}, D_{n}\right\}\right. \\
& \left.+(1+d)\left(S_{1}-c\left(y_{1}-x_{1}\right)\right)\right) \\
& +(1-\lambda) V_{n+1}\left(\left(y_{2}-D_{n}\right)^{+}, p \min \left\{y_{2}, D_{n}\right\}\right. \\
& \left.+(1+d)\left(S_{2}-c\left(y_{2}-x_{2}\right)\right)\right),
\end{aligned}
$$

where the first inequality follows from Lemma 2 and the second inequality follows form the concavity of $V_{n+1}(x, S)$. Hence $V_{n+1}\left(\left(y-D_{n}\right)^{+}, p \min \left\{y, D_{n}\right\}+(1+d)(S-c(y-x))\right)$ is jointly concave in $(y, x, S)$, and so is its expected value. Finally since

$$
\mathbb{C}=\{(x, y): x \geq 0, y \in[x, x+S / c]\}
$$

is a convex set, applying Proposition B-4 of Heyman and Sobel [11] we conclude that $V_{n}(x, S)$ is jointly concave in $x$ and $S$.

We find it convenient to study the value function in terms of $x$ and $R=S+c x$. Define

$\pi_{n}(y, R)=E\left[V_{n+1}\left(\left(y-D_{n}\right)^{+}, p \min \left\{y, D_{n}\right\}+(1+d)(R-c y)\right)\right]$.

Then, the optimality equation (4) can be rewritten, after introducing a new function $\tilde{V}_{n}$, as

$$
\tilde{V}_{n}(x, R)=V_{n}(x, R-c x)=\max _{x \leq y \leq R / c} \pi_{n}(y, R) .
$$

Note that $\pi_{n}(y, R)$ is jointly concave in $(y, R)$. For given $R$, let $y_{n}^{*}(R)$ be the maximizer of the unconstrained optimization problem $\max _{y} \pi_{n}(y, R)$. Then the optimal inventory policy is given in the following result. Its proof follows directly from Lemma 3 hence it is omitted here.

THEOREM 1: When the state is $(x, S)$ at the beginning of period $n$, a capital-dependent base stock inventory policy $y_{n}^{*}(R)$, where $R=S+c x$, is optimal. More specifically,

(i) if $x \leq y_{n}^{*}(R)-S / c$, it is optimal to order up to $R / c$;

(ii) if $y_{n}^{*}(R)-S / c<x<y_{n}^{*}(R)$ then it is optimal to order up to $y_{n}^{*}(R)$;

(iii) if $x \geq y_{n}^{*}(R)$, then it is optimal not to order anything.

We refer to $y_{n}^{*}(R)$ as the optimal base-stock level for period $n$. Hence, for each state $(x, S)$ with $R=S+c x$ there is an order-up-to level $y_{n}^{*}(R)$. Because of the constraint $y \leq R / c$, the base-stock level may not be achieved. The optimal achieved inventory level is $\min \left\{y_{n}^{*}(R), R / c\right\}$ if $x \leq y_{n}^{*}(R)$, and it is $x$ otherwise. This is similar to the inventory control problems with finite supply capacity, for which the optimal strategy is to make the inventory level, within the supply capacity, as close to the order-up-to level as possible. In the following, we let

$$
\hat{y}_{n}^{*}(R)=\min \left\{y_{n}^{*}(R), R / c\right\}
$$

and refer to $\hat{y}_{n}^{*}(R)$ as the optimal replenishment level of period $n$. Therefore, if $x \leq y_{n}^{*}(R)$ then the inventory level at period $n$ is replenished to $\hat{y}_{n}^{*}(R)$, and no order is placed otherwise. Thus, if the state of the system at the beginning of period $n$ is $(x, S)$, then the optimal inventory level for period $n$ after replenishment decision is $\min \left\{\hat{y}_{n}^{*}(S+c x), x\right\}$.

The optimal strategy could still be complicated because $y_{n}^{*}(R)$ is a function of $R$. In the following, we show that for large $R$, the value function $\pi_{n}(y, R)$ can be decoupled into two separate functions of on-hand inventory level $y$ and $R$. This result will simplify the optimal policy significantly for large values of $R$. To that end, we introduce a sequence of concave functions $G_{n}(y)$ as follows: $G_{N+1}(y)=(\gamma-c) y$ and for $n=1, \ldots, N$,

$$
\begin{array}{r}
G_{n}(y)=(1+d)^{N-n}\left((p-c) E\left[\min \left\{y, D_{n}\right\}\right]-d c y\right) \\
+E\left[G_{n+1}\left(\max \left\{a_{n+1}^{*},\left(y-D_{n}\right)^{+}\right\}\right)\right]
\end{array}
$$

where $a_{N+1}^{*}=0$ and for $n=1, \ldots, N, a_{n}^{*}$ is the maximizer of $G_{n}(y)$.

The number $a_{n}^{*}$ will play a central role in determining the optimal control policy. The following result establishes some properties satisfied by $a_{n}^{*}, n=1, \ldots, N$. 
LEMMA 4: The following relationship is satisfied:

$$
\begin{aligned}
F^{-1}\left(\frac{p-(1+d) c}{p-c}\right) \geq a_{1}^{*} & \geq a_{2}^{*} \geq \cdots \geq a_{N}^{*} \\
& =F^{-1}\left(\frac{p-(1+d) c}{p-\gamma}\right),
\end{aligned}
$$

where $F^{-1}$ is the inverse function of $F$.

PROOF: We prove the result by induction. Clearly

$$
\begin{aligned}
G_{N}(y) & =(p-c) E \min \left\{y, D_{N}\right\}-d c y+G_{N+1}\left(\left(y-D_{N}\right)^{+}\right) \\
& =(p-c) E \min \left\{y, D_{N}\right\}-d c y+(\gamma-c)\left(y-D_{N}\right)^{+},
\end{aligned}
$$

is concave in $y$, its maximizer

$$
a_{N}^{*}=F^{-1}\left(\frac{p-(1+d) c}{p-\gamma}\right) \leq F^{-1}\left(\frac{p-(1+d) c}{p-c}\right)
$$

is the newsvendor solution. Assume that we have proved the result for $n+1$, i.e., $G_{n+1}, \ldots, G_{N}$ are concave and

$$
\begin{aligned}
F^{-1}\left(\frac{p-(1+d) c}{p-c}\right) \geq a_{n+1}^{*} \geq & a_{n+2}^{*} \geq \cdots \geq a_{N}^{*} \\
& =F^{-1}\left(\frac{p-(1+d) c}{p-\gamma}\right),
\end{aligned}
$$

we proceed to prove $n$. Taking derivatives of $G_{n}(y)$ with respect to $y$ yields

$$
\begin{aligned}
G_{n}^{\prime}(y)= & (1+d)^{N-n}[(p-c)(1-F(y))-d c] \\
& +E\left[G_{n+1}^{\prime}\left(y-D_{n}\right) 1\left[D_{n} \leq y-a_{n+1}^{*}\right]\right], \\
G_{n}^{\prime \prime}(y)= & -(1+d)^{N-n}(p-c) f(y) \\
& +E\left[G_{n+1}^{\prime \prime}\left(y-D_{n}\right) 1\left[D_{n} \leq y-a_{n+1}^{*}\right]\right] .
\end{aligned}
$$

Hence it follows from the induction assumption that (8) is nonpositive and $G_{n}(y)$ is concave in $y$.

Substituting $y=a_{n+1}^{*}$ in (7), the second term on the right hand side of (7) is 0 , and the first term is nonnegative. Thus $G_{n}^{\prime}\left(a_{n+1}^{*}\right) \geq 0$ and $a_{n}^{*} \geq a_{n+1}^{*}$. Furthermore, note that the first term on the right hand side of (7) vanishes at $y=F^{-1}\left(\frac{p-(1+d) c}{p-c}\right)$, while the second term

$$
\begin{aligned}
G_{n+1}^{\prime}\left(y-D_{n}\right) 1\left[D_{n} \leq\right. & \left.y-a_{n+1}^{*}\right] \\
& =G_{n+1}^{*}\left(y-D_{n}\right) 1\left[y-D_{n} \geq a_{n+1}^{*}\right]
\end{aligned}
$$

is always nonpositive because $a_{n+1}^{*}$ is the maximizer of $G_{n+1}$. This shows that $a_{n}^{*} \leq F^{-1}\left(\frac{p-(1+d) c}{p-c}\right)$.

The value $a_{n}^{*}$ will serve as the ideal order-up-to level for period $n$. We note that, the problem in the final period is essentially a newsvendor problem with capital constraint, its optimal order-up-to level is well-known and is given by the last number in (6). This gives us the lower bound in Lemma 4. The most desirable situation for the firm would be to have the option of returning whatever is left to the supplier at the price paid, $c$, and in this case there would be no risk and the optimal inventory level can be set aggressively, i.e., set the inventory level to the first number in (6). This explains the upper bound in Lemma 4. In general, when there is more period remaining to go, then it is more likely that the on-hand inventory can be successfully used to satisfy future demand, and this explains why the optimal level $a_{n}^{*}$ is decreasing in $n$.

The following decomposition result enables us to obtain an extremely simple form, as well as a computational algorithm, for the optimal replenishment level $\hat{y}_{n}^{*}(R)$. More specifically, we will eventually show that $\hat{y}_{n}^{*}(R)$ can be completely determined by the single parameter, $a_{n}^{*}$.

\section{THEOREM 2:}

(i) For any period $n$, when $R \geq c a_{n+1}^{*}$ and $y \leq R / c$, the objective function can be decomposed as

$$
\pi_{n}(y, R)=(1+d)^{N+1-n} R+G_{n}(y) .
$$

(ii) If $R \geq c a_{n}^{*}$, then the optimal order-up-to level is $y_{n}^{*}(R)=a_{n}^{*}$, and if $R<c a_{n}^{*}$, then $y_{n}^{*}(R) \geq R / c$.

Theorem 2 states that, for large $R$ and small inventory level $y$, the value function $\pi_{n}(y, R)$ can be decomposed to concave functions of $R$ and $y$ alone. This is not true, however, for small $R$ or large $y$. Indeed, in general we would expect the value function $\pi_{n}(y, R)$ to be a complicated function of $(y, R)$, and the separability comes as a surprise. It is this separability result that enables us to significantly simplify the optimal inventory control strategy. Note that $y_{n}^{*}(R)$ is the optimal solution for $\min _{y} \pi_{n}(y, R)$, which is the desired inventory level for stage $n$ without the capital constraint, whereas $a_{n}^{*}$ is a constant that is the maximizer of concave function $G_{n}(\cdot)$. Part (ii) of Theorem 2 states that $y_{n}^{*}(R)$ becomes flat and equal to $a_{n}^{*}$ on $R \geq c a_{n}^{*}$.

The proof of Theorem 2 is lengthy thus it is provided in the Appendix. Intuitively, it says that when the capital level is low, then it is not sufficient to satisfy the desired orderup-to level, or mathematically $y_{n}^{*}(R) \geq R / c$; on the other hand, when the capital level is large, then it does not affect the optimal operational strategy, i.e, the ordering quantity $y_{n}^{*}(R)=a_{n}^{*}$ is a constant. The cutting point for these two regions is $a_{n}^{*}$ which is the maximum point of the concave function $G_{n}$ defined by (5).

The following is the main result of this article. Its proof follows directly from (ii) of Theorem 2 and $\hat{y}_{n}^{*}(R)=$ $\min \left\{y_{n}^{*}(R), R / c\right\}$. 
THEOREM 3: Suppose that the state at the beginning of period $n$ is $(x, S)$ and let $R=S+c x$. The optimal replenishment level for period $n$ is

$$
\hat{y}_{n}^{*}(R)= \begin{cases}a_{n}^{*}, & \text { if } R \geq c a_{n}^{*}, \\ R / c, & \text { if } R \leq c a_{n}^{*}\end{cases}
$$

That is, for any period $n$ with state $(x, R)$, the optimal inventory control policy is to,

(1) replenish the inventory level to $R / c$ if $R / c \leq a_{n}^{*}$;

(2) replenish the inventory level to $a_{n}^{*}$ if $x<a_{n}^{*}<R / c$; and

(3) do not order anything if $x \geq a_{n}^{*}$.

Therefore, for each period $n$, the optimal replenishment level first linearly increases with the wealth level $R$ at rate $1 / c$ until $c a_{n}^{*}$ and then it becomes flat from $R=c a_{n}^{*}$. This gives us an exceedingly simple inventory control policy: The inventory control policy is determined solely by a capitalindependent level $a_{n}^{*}$, at the beginning of period $n$, the firm replenishes its inventory level to $a_{n}^{*}$ as long as there is sufficient capital available; if there is no sufficient capital, then it replenishes as much as possible - that is, it uses up all of its capital.

The optimal replenishment level $\hat{y}_{n}^{*}(R)$ is determined by a single parameter $a_{n}^{*}$, and the computation of $a_{n}^{*}$ is straightforward. A nested algorithm is summarized as follows.

\section{Algorithm:}

Step 1. Set $a_{N+1}^{*}=0$, and compute $G_{N}$ by (5). Set $n=N$.

Step 2. Computed $a_{n}^{*}$ via concave function $G_{n}(y)$ of (5). Step 3. If $n=1$ then stop. Otherwise set $n:=n-1$ and repeat Step 2.

REMARK 1: Note that, though the optimal base-stock level $y_{n}^{*}(R)$ is a constant $a_{n}^{*}$ on $R \geq c a_{n}^{*}$, it can be a very complicated function on $R \leq c a_{n}^{*}$. As a matter of fact, $y_{n}^{*}(R)$ may not be even monotone on $R \in\left[0, c a_{n}^{*}\right]$. See the numerical example at the end of this section. Nevertheless, Theorem 3 states that this does not complicate the optimal replenishment level $\hat{y}_{n}^{*}(R)$ : On $R \leq c a_{n}^{*}$, the optimal replenishment level is $R / c$, i.e., simply use up all the capital. Hence, in implementing the optimal policy, the complicated optimal-up-to level $y_{n}^{*}(R)$ on the range $R \leq c a_{n}^{*}$ is not used.

The following theorem presents the comparative statics results for the optimal policy on the selling price $p$, purchasing price $c$, salvage value $\gamma$, and interest rate $d$.

\section{THEOREM 4:}

(i) The optimal control policy parameters $a_{n}^{*}, n=$ $1, \ldots, N$, are increasing in $\gamma$ and $p$, and decreasing in $c$ and $d$.

(ii) As $\gamma$ increases from $-\infty$ to $c, a_{n}^{*}$ increases from 0 to $F^{-1}\left(\frac{p-(1-d) c}{p-c}\right)$. In particular, as $\gamma=c$, the optimal inventory policy is the same for each period and is given by

$$
a_{1}^{*}=a_{2}^{*}=\cdots=a_{N}^{*}=F^{-1}\left(\frac{p-(1+d) c}{p-c}\right)
$$

PROOF: (i) We first prove the result on $\gamma, p$, and $c$. Because $a_{n}^{*}$ is the maximizer of $G_{n}(y)$, it suffices to prove $G_{n}^{\prime}(y)$ is increasing in $\gamma$ and $p$, and decreasing in $c$.

By induction. First notice that

$$
G_{N}^{\prime}(y)=[(p-c)(1-F(y))-d c]+\int_{0}^{v}(\gamma-c) d F(z),
$$

which is clearly increasing in $\gamma$ and $p$, and decreasing in $c$. Suppose $G_{n+1}^{*}$ is increasing in $\gamma$ and $p$ and decreasing in $c$, then we have

$$
\begin{aligned}
\frac{\partial G_{n}^{\prime}(y)}{\partial \gamma}= & \int_{0}^{\left(y-a_{n+1}^{*}\right)^{+}} \frac{\partial G_{n+1}^{\prime}(y-z)}{\partial \gamma} d F(z), \\
\frac{\partial G_{n}^{\prime}(y)}{\partial p}= & (1+d)^{N-n} p(1-F(y)) \\
& +\int_{0}^{\left(y-a_{n+1}^{*}\right)^{+}} \frac{\partial G_{n+1}^{\prime}(y-z)}{\partial p} d F(z), \\
\frac{\partial G_{n}^{\prime}(y)}{\partial c}= & -(1+d)^{N-n}(c(1-F(y))+d) \\
& +\int_{0}^{\left(y-a_{n+1}^{*}\right)^{+}} \frac{\partial G_{n+1}^{\prime}(y-z)}{\partial \gamma} d F(z) .
\end{aligned}
$$

Hence $\partial G_{n}^{\prime}(y) / \partial \gamma \geq 0, \partial G_{n}^{\prime}(y) / \partial p \geq 0$ and $\partial G_{n}^{\prime}(y) / \partial c \leq$ 0 follow immediately from the induction hypothesis.

To prove $a_{n}^{*}$ is decreasing in $d$, by Lemma 4 it suffices to show that $\partial G_{n}^{\prime}(y) / \partial d \leq 0$ for $n=1, \ldots, N$ on the range

$$
y \geq F^{-1}\left(\frac{p-(1+d) c}{p-\gamma}\right) .
$$

This is again proved by induction and it is trivially true for $N$. Suppose it has been established for $n+1$. Then, on range 


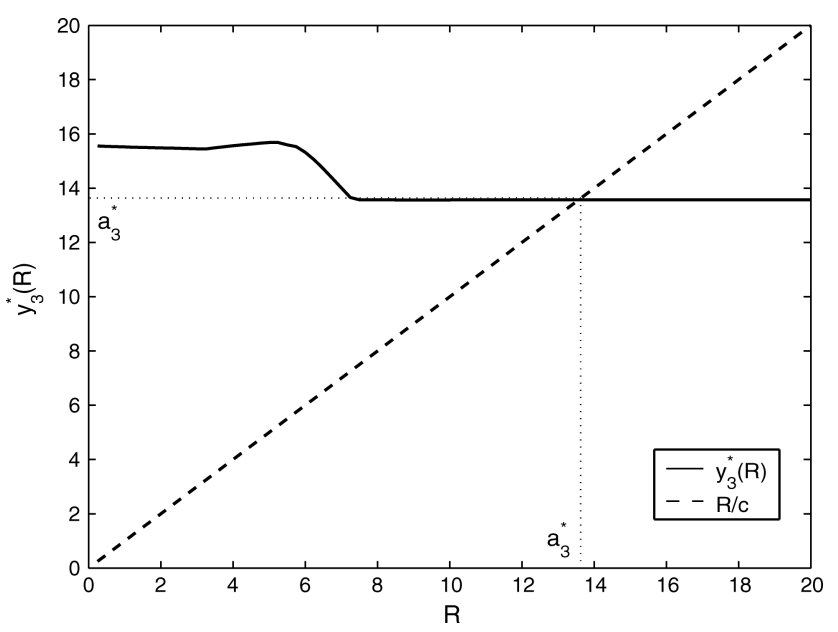

Figure 1. Optimal base stock policy for period 3.

(9) we have

$$
\begin{aligned}
\frac{\partial G_{n}^{\prime}(y)}{\partial d}= & (1+d)^{N-n-1}[(p-c)(1-F(y))-d c \\
& -(1+d) c]+\int_{0}^{\left(y-a_{n+1}^{*}\right)^{+}} \frac{\partial G_{n+1}^{\prime}(y-z)}{\partial d} d F(z) \\
\leq & (1+d)^{N-n-1}[(p-c)(1-F(y)) \\
& -d c-(1+d) c] \\
\leq & (1+d)^{N-n-1}\left[(p-c) \frac{(1+d) c-\gamma}{p-\gamma}\right. \\
& -d c-(1+d) c] \\
= & \frac{(1+d)^{N-n-1}}{p-\gamma}[-(c-\gamma)(1+d) c \\
& -(p-c) \gamma-(p-\gamma) d c] \\
\leq & 0,
\end{aligned}
$$

where the first inequality follows from induction hypothesis, the second inequality follows from (9), and the last inequality follows from (1).

(ii) If $\gamma=c$, then it follows from Lemma 4 that all $a_{n}^{*}$ are equal, completing the proof of Theorem 4.

As mentioned earlier, the number $a_{n}^{*}$ is basically the ideal order-up-to level for period $n$. When the salvage value or the selling price is higher, it is more profitable to keep a higher inventory thus $a_{n}^{*}$ is increasing in $\gamma$ and $p$. The same argument shows that when the purchasing price $c$ is higher, it is better to reduce the inventory level thus $a_{n}^{*}$ is decreasing in $c$. When $d$ increases, savings account becomes a more attractive option hence the firm will be willing to invest more in the banking account than in the inventory, explaining why $a_{n}^{*}$ is decreasing in $d$. Finally, when the salvage value is the

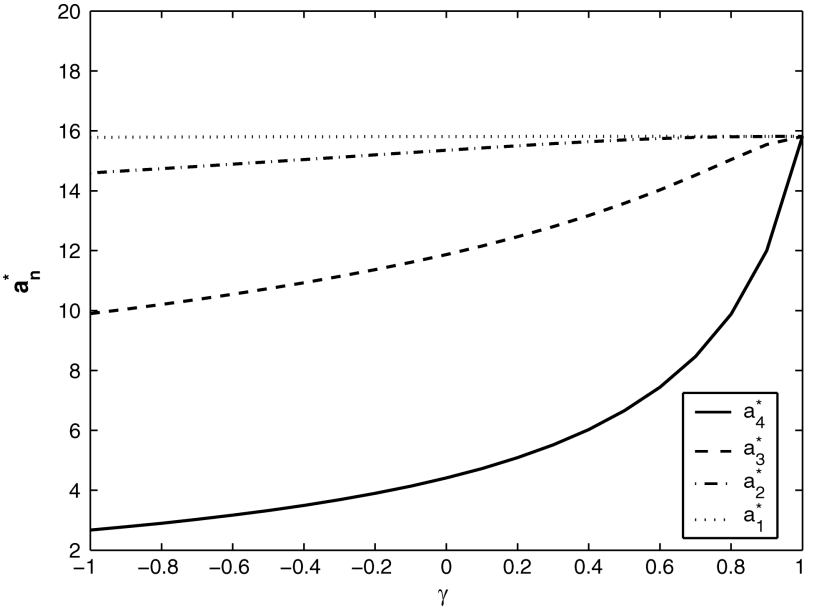

Figure 2. The optimal control strategy $a_{n}^{*}$ on $\gamma$.

same as the ordering cost, then there is no risk associated with salvaging the inventory at the end. Therefore, the problem in each period is a newsvendor problem with capital constraint. This explains part (ii) of Theorem 4.

We present numerical examples to demonstrate the optimal inventory policy and its dependency on wealth level $R$, salvage value $\gamma$, and interest rate $d$. The model parameters in all these numerical examples are $p=1.3$ and $c=1$. In Fig. 1 the interest rate is set at $d=0.1$ and the savage value is $\gamma=0.5$; in Fig. 2 the interest rate is set at $d=0.1$; and in Fig. 3 , the savage value is $\gamma=0.5$. The demand has truncated normal distribution with mean 10 and variance 10. Assume there are totally $N=4$ periods.

First compute the optimal policy parameters $a_{n}^{*}$ for $n=$ $1,2,3$, and 4 via optimizing concave functions $G_{n}(y)$. Notice that the upper and lower bounds for $a_{n}^{*}$ are given by

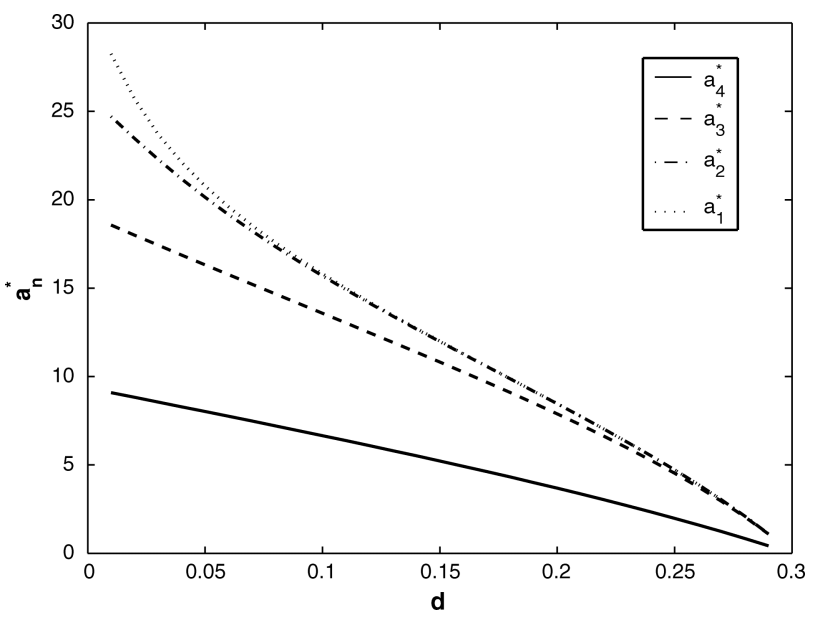

Figure 3. The optimal control strategy $a_{n}^{*}$ on $d$. 
$F^{-1}\left(\frac{p-(1+d) c}{p-c}\right)=15.8151$ and $F^{-1}\left(\frac{p-(1+d) c}{p-\gamma}\right)=6.6547$. Figure 1 demonstrates how the optimal inventory policy depends on the wealth level $R$. The numerical results are for period 3. It shows that when $R \geq c a_{3}^{*}, y_{3}^{*}(R)=a_{3}^{*}$; and when $R<\mathrm{ca}_{3}^{*}, y_{3}^{*}(R)>R / c$. Note that the minimum of the dotted line and the solid curve is $\hat{y}_{3}^{*}(R)$.

It is interesting to observe that the optimal order-up-to level $y_{3}^{*}(R)$ is complicated and is not even monotone in $R$ on the range $R \leq c a_{3}^{*}$. The optimal replenishment level $\hat{y}_{n}^{*}(R)$ is, however, always extremely simple, as we noted earlier.

Figure 2 presents the optimal policy in terms of savage value $\gamma$ on the range $[-1,1]$. It demonstrates that $a_{n}^{*}$ is decreasing in $n$ and increasing in $\gamma$.

Figure 3 presents the optimal policy in terms of interest rate $d$ on the range $[0,0.3]$. It demonstrates that $a_{n}^{*}$ is decreasing in $n$ and $d$.

\section{DISCUSSION}

In this article, we study a dynamic inventory control problem with financial constraints. We derive the optimal inventory policy for each period, and characterize the dependence of the firm's optimal operational policy on its financial status. We also analyze the relationship between the optimal control parameters and system parameters.

Many interesting issues remain to be investigated. For example, if there is a holding cost rate $h$ and shortage cost rate $b$ for each period, then the optimality equation becomes

$$
\max _{y_{1}, \ldots, y_{N}} E\left[S_{N+1}\right]
$$

subject to

$$
0 \leq y_{n}-x_{n} \leq S_{n} / c, \quad n=1,2, \ldots, N,
$$

where

$$
\begin{aligned}
S_{n+1}= & p \min \left\{y_{n}, D_{n}\right\}-h \max \left\{y_{n}-D_{n}, 0\right\} \\
& -b \max \left\{D_{n}-y_{n}, 0\right\}+(1+d)\left(S_{n}-c\left(y_{n}-x_{n}\right)\right),
\end{aligned}
$$

and, as before, $x_{n+1}=\left(y_{n}-D_{n}\right)^{+}$. Note that Lemmas 1 , 2, and 3 continue to hold, thus Theorem 1 also holds and the optimal inventory control policy is a capital dependent base-stock policy. As a matter of fact, Theorem 1, as well as Lemmas 1, 2, and 3 hold true under much more general settings, e.g., under general revenue function, and under utility function optimization, etc. However, for Theorems 2 and 3, the objective function can no longer be decomposed, and the control parameters of the optimal base-stock policy are complicated and state-dependent. The problem will be even more complicated if we allow the selling price $p$ to be a decision variable when the demand $D_{n}$ depends on the selling price in period $n$.

The setting used in this article assumes that the demands over periods are independent and identically distributed. We point out that the results Lemmas 1-3 and Theorem 1 hold true as long as the demands over periods are independent and they do not need to be identically distributed, and other results can be extended too to the non-identically distributed demand case.

The firm considered in this article is self-financed, a natural extension of the model is to allow the firm to borrow from the bank or other lenders. One can even impose an upper borrowing limit, which is typical in many applications. Some other interesting issues to consider include delayed payment, and risk-averse retailers. We find that for most such extensions, the results in Lemmas 1-3 and Theorem 1 can still be obtained. However, more precise structure of the optimal control policies beyond the "capital-dependent base-stock," such as Theorem 3 in this article, is difficult to obtain without imposing further structure in the model. These are just a few possible extensions and it appears that each of these variations will lead to different optimal solution structure that is worthy of study.

\section{APPENDIX: PROOF OF THEOREM 2} have

The proof is by induction. By the definition of $G_{n}(y)$ and Lemma 4 we $\pi_{N}(y, R)=p E \min \left\{y, D_{N}\right\}+(1+d)(R-c y)+\gamma E\left(y-D_{N}\right)^{+}$ $=(1+d) R+G_{N}(y)$

hence $y_{N}^{*}(R)=a_{N}^{*}$ for all $R$.

Assume that the results have been proved for $n+1$, i.e., when $R \geq c a_{n+2}^{*}$ and $y \leq R / c, \pi_{n+1}(y, R)$ can be decomposed as

$$
\pi_{n+1}(y, R)=(1+d)^{N-n} R+G_{n+1}(y)
$$

and that if $R \leq c a_{n+1}^{*}$, then $y_{n+1}^{*}(R) \geq R / c$; if $R \geq c a_{n+1}^{*}$, then $y_{n+1}^{*}(R)=a_{n+1}^{*}$.

To simplify the proof it is convenient to define a new function

$$
\begin{aligned}
\tilde{V}_{n}(x, R)= & V_{n}(x, S) \\
= & V_{n}(x, R-c x) \\
= & \max _{x \leq y \leq R / c} \pi_{n}(y, R) \\
= & \max _{x \leq y \leq R / c} E\left[\tilde { V } _ { n + 1 } \left(\left(y-D_{n}\right)^{+},(p-c) \min \left\{y, D_{n}\right\}\right.\right. \\
& +(1+d) R-d c y)] .
\end{aligned}
$$

From Lemma 3 it is straightforward to prove that $\tilde{V}_{n}(x, R)$ is jointly concave in $x$ and $R$. From Theorem 1 we have

$$
\tilde{V}_{n+1}(x, R)= \begin{cases}\pi_{n+1}(R / c, R), & R / c \leq y_{n+1}^{*}(R) \\ \pi_{n+1}\left(y_{n+1}^{*}(R), R\right), & x<y_{n+1}^{*}(R)<R / c \\ \pi_{n+1}(x, R), & x \geq y_{n+1}^{*}(R)\end{cases}
$$

Naval Research Logistics DOI 10.1002/nav 
On the other hand, by the induction assumption and Lemma 4, the following observations are made:

(a) If $R \leq c a_{n+2}^{*} \leq c a_{n+1}^{*}$, then $y_{n+1}^{*}(R) \geq R / c$, hence $\tilde{V}_{n+1}(x, R)=$ $\pi_{n+1}(R / c, R)$.

(b) If $c a_{n+2}^{*}<R \leq c a_{n+1}^{*}$, then $y_{n+1}^{*}(R) \geq R / c$ and $\pi_{n+1}(y, R)$ can be decomposed as (10), and hence

$$
\tilde{V}_{n+1}(x, R)=(1+d)^{N-n} R+G_{n+1}(R / c) .
$$

(c) If $c x<c a_{n+1}^{*}<R$, then $y_{n+1}^{*}(R)=a_{n+1}^{*}$ and hence $x<$ $y_{n+1}^{*}(R)<R / c$. In this case $\pi_{n+1}(y, R)$ can be decomposed as (10), thus

$$
\tilde{V}_{n+1}(x, R)=(1+d)^{N-n} R+G_{n+1}\left(a_{n+1}^{*}\right) .
$$

(d) If $a_{n+1}^{*} \leq x \leq R / c$, then $y_{n+1}^{*}(R)=a_{n+1}^{*}$ and hence $x \geq$ $y_{n+1}^{*}(R)$. Further $\pi_{n+1}(y, R)$ can be decomposed by (10) as

$$
\tilde{V}_{n+1}(x, R)=(1+d)^{N-n} R+G_{n+1}(x) .
$$

The structure of the proof is divided into the following two propositions.

PROPOSITION 1: If $R \geq c a_{n+1}^{*}$ and $y \leq R / c$, then

$$
\pi_{n}(y, R)=(1+d)^{N+1-n} R+G_{n}(y) .
$$

PROOF: We can rewrite (11) as

$\tilde{V}_{n+1}(x, R)= \begin{cases}\pi_{n+1}(R / c, R), & R \leq c a_{n+2}^{*} \\ (1+d)^{N-n} R+G_{n+1}(R / c), & c a_{n+2}^{*}<R \leq c a_{n+1}^{*} \\ (1+d)^{N-n} R+G_{n+1}\left(a_{n+1}^{*}\right), & c x<c a_{n+1}^{*}<R \\ (1+d)^{N-n} R+G_{n+1}(x), & x \geq a_{n+1}^{*}\end{cases}$

The last two cases show that if $R \geq c a_{n+1}^{*}, \tilde{V}_{n+1}(x, R)$ can be rewritten as

$$
\tilde{V}_{n+1}(x, R)=(1+d)^{N-n} R+G_{n+1}\left(\max \left\{a_{n+1}^{*}, x\right\}\right) .
$$

As a result, if $R \geq c a_{n+1}^{*}$ and $y \leq R / c$, then $(p-c) \min \left\{y, D_{n}\right\}+(1+$ d) $R-d c y \geq c a_{n+1}^{*}$, and hence $\pi_{n}(y, R)$ can be expressed as

$$
\begin{aligned}
\pi_{n}(y, R)= & E\left[\tilde { V } _ { n + 1 } \left(\left(y-D_{n}\right)^{+},(p-c) \min \left\{y, D_{n}\right\}\right.\right. \\
& +(1+d) R-d c y)] \\
= & (1+d)^{N+1-n} R+(1+d)^{N-n}((p-c) \\
& \left.\times E \min \left\{y, D_{n}\right\}-d c y\right) \\
& +E G_{n+1}\left(\max \left\{a_{n+1}^{*},\left(y-D_{n}\right)^{+}\right\}\right) \\
= & (1+d)^{N+1-n} R+G_{n}(y) .
\end{aligned}
$$

Therefore, when $R \geq c a_{n+1}^{*}$ and $y \leq R / c$, the maximizer of $\pi_{n}(y, R)$, $y_{n}^{*}(R)$, is equal to $a_{n}^{*}$, the maximizer of $G_{n}(y)$. Furthermore, when $R \geq$ $c a_{n}^{*} \geq c a_{n+1}^{*}, y_{n}^{*}(R)=a_{n}^{*}$, and when $c a_{n+1}^{*}<R<c a_{n}^{*}, y_{n}^{*}(R)=a_{n}^{*}>$ $R / c$.

PROPOSITION 2: If $R \leq c a_{n+1}^{*}$, then $y_{n}^{*}(R) \geq R / c$.

PROOF: For notational convenience in what follows we use $\tilde{V}_{n, 1}(x, R)$ and $\tilde{V}_{n, 2}(x, R)$ to represent the partial derivatives with respect to $x$ and $R$ Naval Research Logistics DOI 10.1002/nav respectively, and $\tilde{V}_{n, 12}(x, R)$ the cross derivative. From (12), taking partial derivatives of $\tilde{V}_{n+1}(x, R)$ yields

$$
\tilde{V}_{n+1,1}(x, R)= \begin{cases}0, & x<a_{n+1}^{*} \\ G_{n+1}^{\prime}(x), & x \geq a_{n+1}^{*}\end{cases}
$$

and

$$
\tilde{V}_{n+1,2}(x, R)= \begin{cases}d \pi_{n+1}(R / c, R) / d R, & R \leq c a_{n+2}^{*} \\ (1+d)^{N-n}+G_{n+1}^{\prime}(R / c) / c, & c a_{n+2}^{*}<R \leq c a_{n+1}^{*} \\ (1+d)^{N-n}, & R>c a_{n+1}^{*}\end{cases}
$$

Note that $\tilde{V}_{n+1,2}(x, R)$ is independent of $x$, hence $\tilde{V}_{n+1,12}(x, R)=0$. By

$$
\begin{aligned}
\pi_{n}(y, R)=E\left[\tilde { V } _ { n + 1 } \left(\left(y-D_{n}\right)^{+},(p-c) \min \left\{y, D_{n}\right\}\right.\right. & \\
& +(1+d) R-d c y)]
\end{aligned}
$$

taking derivative of $\pi_{n}(y, R)$ with respect to $y$ yields

$$
\begin{aligned}
\pi_{n, 1}(y, R)= & \int_{0}^{y}\left[\tilde{V}_{n+1,1}(y-z,(p-c) z+(1+d) R-d c y)\right. \\
& -d c \tilde{V}_{n+1,2}(y-z,(p-c) z \\
& +(1+d) R-d c y)] d F(z) \\
& +(p-(1+d) c)(1-F(y)) \tilde{V}_{n+1,2} \\
& \times(0,(p-(1+d) c) y+(1+d) R)
\end{aligned}
$$

Because $y_{n}^{*}(R)$ is the maximizer of $\pi_{n}(y, R)$, to prove $y_{n}^{*}(R) \geq R / c$ when on $R \leq c a_{n+1}^{*}$, it suffices to prove $\pi_{n, 1}(R / c, R) \geq 0$ on $R \leq c a_{n+1}^{*}$. Noting $\tilde{V}_{n, 1}(x, R)=0$ when $x \leq R / c \leq a_{n+1}^{*}$, we have

$$
\begin{aligned}
\pi_{n, 1}(R / c, R)= & (p-(1+d) c)(1-F(R / c)) \tilde{V}_{n+1,2}(0, p R / c) \\
& -d c \int_{0}^{R / c} \tilde{V}_{n+1,2}(R / c-z, \quad(p-c) z+R) d F(z) \\
\geq & (p-(1+d) c)(1-F(R / c)) \tilde{V}_{n+1,2}(0, p R / c) \\
& -d c F(R / c) \tilde{V}_{n+1,2}(R / c, R),
\end{aligned}
$$

where the inequality follows from the concavity of $\tilde{V}_{n+1}(x, R)$ in $R$ :

$$
\tilde{V}_{n+1,2}(R / c-z,(p-c) z+R) \leq \tilde{V}_{n+1,2}(R / c-z, R)
$$

and that $\tilde{V}_{n+1,12}(x, R)=0$ is independent of $x$ :

$$
\tilde{V}_{n+1,2}(R / c-z, R)=\tilde{V}_{n+1,2}(R / c, R) .
$$

Since $R \leq c a_{n+1}^{*} \leq c F^{-1}\left(\frac{p-(1+d) c}{p-c}\right)$, we have

$$
(p-(1+d) c)(1-F(R / c)) \geq d c F(R / c) .
$$

To prove that the right hand side of (16) is nonnegative, we consider two ranges of $R$ separately.

CASE 1: $c a_{n+2}^{*}<R \leq c a_{n+1}^{*}$. By (7) we have

$$
G_{n+1}^{\prime}(y) \leq(1+d)^{N-n-1}((p-c)(1-F(y))-d c
$$

and since $\tilde{V}_{n+1,2}^{\prime}(x, R)$ is decreasing in $R$, it follows from (14) that

$$
\tilde{V}_{n+1,2}(0, p R / c) \geq \lim _{y \rightarrow \infty} \tilde{V}_{n+1,2}(0, y) \geq(1+d)^{N-n}
$$


Hence applying these inequalities and (14) on the interval $c a_{n+2}^{*}<R$ $\leq c a_{n+1}^{*}$ yields

$$
\begin{aligned}
&(p-(1+d) c)(1-F(R / c)) \tilde{V}_{n+1,2}(0, p R / c)-d c F(R / c) \tilde{V}_{n+1,2}(R / c, R) \\
& \geq(p-(1+d) c)(1-F(R / c))(1+d)^{N-n} \\
&-d c F(R / c)\left[(1+d)^{N-n}+G_{n+1}^{\prime}(R / c) / c\right] \\
& \geq {[(p-(1+d) c)(1-F(R / c))-d c F(R / c)](1+d)^{N-n} } \\
&-d c F(R / c)(1+d)^{N-n-1}[(p-c)(1-F(R / c))-d c] / c \\
&=(1+d)^{N-1-n}[1+d(1-F(R / c))] \\
& \times[(p-(1+d) c)(1-F(R / c))-d c F(R / c)] \\
& \geq 0,
\end{aligned}
$$

where the last inequality follows from (17). Therefore, $\pi_{n, 1}(R / c, R) \geq 0$ in this case.

CASE 2: $R \leq c a_{n+2}^{*}$. From (18), we have

$$
\begin{aligned}
& \pi_{n+1}(R / c, R)=E\left[\tilde { V } _ { n + 2 } \left(\left(R / c-D_{n+1}\right)^{+},\right.\right. \\
& \left.\left.(p-c) \min \left\{R / c, D_{n+1}\right\}+R\right)\right],
\end{aligned}
$$

hence

$$
\begin{aligned}
d \pi_{n+1}(R / c, R) / d R= & \int_{0}^{R / c}\left[\tilde{V}_{n+2,1}(R / c-z,(p-c) z+R)\right] d F(z) \\
& +\frac{p}{c}(1-F(R / c)) \tilde{V}_{n+2,2}(0, p R / c) \\
& +\int_{0}^{R / c}\left[\tilde{V}_{n+2,2}(R / c-z,(p-c) z+R)\right] d F(z) \\
= & \frac{p}{c}(1-F(R / c)) \tilde{V}_{n+2,2}(0, p R / c) \\
& +\int_{0}^{R / c}\left[\tilde{V}_{n+2,2}(R / c-z,(p-c) z+R)\right] d F(z) \\
\leq & \frac{p}{c}(1-F(R / c)) \tilde{V}_{n+2,2}(0, p R / c) \\
& +\frac{p-(1+d) c}{d c}(1-F(R / c)) \tilde{V}_{n+2,2}(0, p R / c) \\
= & \frac{(p-c)(1+d)}{d c}(1-F(R / c)) \tilde{V}_{n+2,2}(0, p R / c),
\end{aligned}
$$

where the second equality follows from $\left.\tilde{V}_{n+2,1}(R / c-z,(p-c) z+R)\right]=0$ because of (13) and the induction assumptions for $n+2$, and the inequality is based on the following argument. When $R \leq c a_{n+2}^{*}<c a_{n+1}^{*}$, from the induction assumption we have $y_{n+1}^{*}(R) \geq R / c$, and hence $\pi_{n+1,1}(R / c, R) \geq$ 0 , which implies, by (16) for $n+1$, that

$$
\begin{aligned}
& (p-(1+d) c)(1-F(R / c)) \tilde{V}_{n+2,2}(0, p R / c) \\
& \quad \geq d c \int_{0}^{R / c} \tilde{V}_{n+2,2}(R / c-z,(p-c) z+R) d F(z) .
\end{aligned}
$$

For $R \leq c a_{n+2}^{*}$, applying (16) and (19) we obtain

$$
\begin{aligned}
\pi_{n, 1}(R / c, R) \geq & (p-(1+d) c)(1-F(R / c)) \tilde{V}_{n+1,2}(0, p R / c) \\
& -d c F(R / c) \tilde{V}_{n+1,2}(R / c, R) \\
= & (p-(1+d) c)(1-F(R / c)) \tilde{V}_{n+1,2}(0, p R / c) \\
& -d c F(R / c) d \pi_{n+1}(R / c, R) / d R \\
\geq & (1-F(R / c))\left[(p-(1+d) c) \tilde{V}_{n+1,2}(0, p R / c)\right. \\
& \left.-(p-c)(1+d) F(R / c) \tilde{V}_{n+2,2}(0, p R / c)\right] \\
\geq & (p-(1+d) c)(1-F(R / c))\left[\tilde{V}_{n+1,2}(0, p R / c)\right. \\
& \left.-(1+d) \tilde{V}_{n+2,2}(0, p R / c)\right],
\end{aligned}
$$

where the last inequality follows from $(p-c) F(R / c) \leq p-(1+d) c$ because of $R \leq c a_{n+1}^{*}<c F^{-1}\left(\frac{p-(1+d) c}{p-c}\right)$.

Therefore, the desired result $\pi_{n, 1}(R / c, R) \geq 0$ will follow if we can prove

$$
\tilde{V}_{n+1,2}(0, R)-(1+d) \tilde{V}_{n+2,2}(0, R) \geq 0
$$

for all $R$. This is again done by backward induction. First we have

$$
\tilde{V}_{N, 2}(0, R)-(1+d) \tilde{V}_{N+1,2}(0, R) \geq(1+d)-(1+d)=0 .
$$

Assume $\tilde{V}_{n+2,2}(0, R)-(1+d) \tilde{V}_{n+3,2}(0, R) \geq 0$, we proceed to prove (20). If $R>c a_{n+2}^{*}$, then by (14) for $n+2$,

$$
\tilde{V}_{n+1,2}(0, R)-(1+d) \tilde{V}_{n+2,2}(0, R)
$$

$$
\geq(1+d)^{N-n}-(1+d)(1+d)^{N-n-1}=0 .
$$

If $c a_{n+3}^{*} \leq R \leq c a_{n+2}^{*}$, then by (7) for $n+2$, we have

$$
G_{n+2}^{\prime}(R / c) \leq(1+d)^{N-n-2}[(p-c)(1-F(R / c))-d c],
$$

and by the concavity of $G(x, R)$ in $R$ we have

$$
\tilde{V}_{n+2,2}(R / c-z,(p-c) z+R) \geq \tilde{V}_{n+2,2}(R / c-z, p R / c),
$$

and since $\tilde{V}_{n+2,2}(x,(p-c) z+R)$ is independent of $x$ when $x \leq c a_{n+2}^{*}$, we have

$$
\tilde{V}_{n+2,2}(R / c-z, p R / c)=\tilde{V}_{n+2,2}(0, p R / c) .
$$

Applying (14) we obtain

$$
\begin{aligned}
\tilde{V}_{n+1,2} & (0, R)-(1+d) \tilde{V}_{n+2,2}(0, R) \\
= & \frac{d \pi_{n+1}(R / c, R)}{d R}-(1+d)^{N-n}-(1+d) G_{n+2}^{\prime}(R / c) / c \\
\geq & \frac{p}{c}(1-F(R / c)) \tilde{V}_{n+2,2}(0, p R / c) \\
& +\int_{0}^{R / c} \tilde{V}_{n+2,2}(R / c-z,(p-c) z+R) d F(z) \\
& -(1+d)^{N-n}-(1+d)^{N-n-1}[(p-c)(1-F(R / c))-d c] / c \\
\geq & {\left[\frac{p}{c}(1-F(R / c))+F(R / c)\right] \tilde{V}_{n+2,2}(0, p R / c) } \\
& -(1+d)^{N-n-1}\left[\frac{p}{c}(1-F(R / c))+F(R / c)\right] \\
= & {\left[\frac{p}{c}(1-F(R / c))+F(R / c)\right]\left(\tilde{V}_{n+2,2}(0, p R / c)-(1+d)^{N-n-1}\right) } \\
\geq & 0,
\end{aligned}
$$


where the first inequality follows from (19) and (21), the second inequality is due to (22) and (23), and the last inequality follows from the observation that, by (14) for $n+2$, when $p R / c \geq R \geq c a_{n+3}^{*}$, we have $\tilde{V}_{n+2,2}(0, p R / c) \geq(1+d)^{N-n-1}$.

Finally, if $R<c a_{n+3}^{*}$, then

$$
\begin{aligned}
\tilde{V}_{n+1,2} & (0, R)-(1+d) \tilde{V}_{n+2,2}(0, R) \\
= & \frac{d \pi_{n+1}(R / c, R)}{d R}-(1+d) \frac{d \pi_{n+2}(R / c, R)}{d R} \\
= & \frac{p}{c}(1-F(R / c))\left[\tilde{V}_{n+2,2}(0, p R / c)-(1+d) \tilde{V}_{n+3,2}(0, p R / c)\right] \\
& +\int_{0}^{R / c}\left[\tilde{V}_{n+2,2}(R / c-z,(p-c) z+R)-(1+d) \tilde{V}_{n+3,2}\right. \\
& \times(R / c-z,(p-c) z+R)] d F(z) \\
\geq & 0
\end{aligned}
$$

where the first equality follows from (14), the second equality follows from (19) for $n+2$ and $n+3$, and the inequality follows from the induction assumption.

Hence we have proved $\pi_{n, 1}(R / c, R) \geq 0$, implying $y_{n}^{*}(R) \geq R / c$ if $R \leq c a_{n+1}^{*}$, completing the proof of Proposition 2.

Theorem 2 follows directly from Propositions 1 and 2.

\section{ACKNOWLEDGMENTS}

The authors are grateful to the Associate Editor and three anonymous referees for detailed comments and suggestions, which have helped improve the exposition of the paper. They also wish to thank Volodymyr (Vlad) Babich for his helpful comments on the manuscript. This work was done while the first author was affiliated with the Department of Industrial and Systems Engineering at North Carolina State University, and his research is partially supported by the NSF under CMMI-0800004.

\section{REFERENCES}

[1] T.W. Archibald, L.C. Thomas, J.M. Betts, and R.B. Johnston, Should start-up companies be cautious? Inventory policies which maximise survival probabilities, Management Sci 48 (2002), 1161-1174.

[2] S. Axsäter, Inventory control, Kluwer Academic Publishers, Norwell, MA, 2000.
[3] V. Babich and M.J. Sobel, Pre-IPO operational and financial decisions, Management Sci 50 (2004), 935-948.

[4] J.R. Birge, Option methods for incorporating risk into linear capacity planning models, Manuf Service Oper Management 2 (2000), 19-31.

[5] D. Bradley, Lack of financial and location planning causes small business bankruptcy, University of Central Arkansas, Conway, AR, 2000.

[6] J.A. Buzacott and R.Q. Zhang, Inventory management with asset-based financing, Management Sci 50 (2004), 1274-1292.

[7] J.B. Chen and W. Zhang, The Report of China's Industrial Development in 2001, Development and Research Center of the State Council, P. R. China, Report Number 2001-181-185, 2001.

[8] F.W. Ciarallo, R. Akella, and T.E. Morton, A periodic review, production planning model with uncertain capacity and uncertain demand - optimality of extended myopic policies, Management Sci 40 (1994), 320-332.

[9] A. Federgruen and P. Zipkin, An inventory model with limited production capacity and uncertain demands. I. The average-cost criterion, Math Oper Res 11 (1986), 193-207.

[10] A. Federgruen and P. Zipkin, An inventory model with limited production capacity and uncertain demands. II. The discounted-cost criterion, Math Oper Res 11 (1986), 208-215.

[11] D.P. Heyman and M.J. Sobel, Stochastic models in operations research, Vol. II: Stochastic optimization, McGraw-Hill, NY, 1984.

[12] Q.H. Hu and M.J. Sobel, Capital structure and inventory management, Working Paper, Weatherhead School of Management, Case Western Reserve University, Cleveland, $\mathrm{OH}$, 2005.

[13] L. Li, M. Shubik, and M.J. Sobel, Production with dividends and default penalties, Working Paper, Weatherhead School of Management, Case Western Reserve University, Cleveland, $\mathrm{OH}, 1997$.

[14] S. Nahmias, Production and operations analysis, 4th ed., McGraw-Hill/Irwin, Boston, 2001.

[15] E.L. Porteus, Foundations of stochastic inventory theory, Stanford University Press, Stanford, CA, 2002.

[16] M.J. Rosenblatt and U.G. Rothblum, On the single resource capacity problem for multi-item inventory systems, Oper Res 38 (1990), 686-693.

[17] Y. Wang and Y. Gerchak, Periodic review production models with variable capacity, random yield, and uncertain demand, Management Sci 42 (1996), 130-137.

[18] Y. Xu and J.R. Birge, Joint proudction and financing decisions: Modeling and analysis, Working paper, Northwestern Univeristy, Evanston, IL, 2004.

[19] P.H. Zipkin, Foundations of inventory management, McGrawHill, Boston, MA, 2000. 